\title{
Macroalgas, microalgas y cianobacterias epífitas del pasto marino Thalassia testudinum (Tracheophyta: Alismatales) en Veracruz y Quintana Roo, Atlántico mexicano
}

Macroalgae, microalgae and cyanobacteria epiphytic of the seagrass Thalassia testudinum (Tracheophyta: Alismatales) in Veracruz and Quintana Roo, Mexican Atlantic

\author{
Rocio Nava-Olvera ${ }^{1 *}$, Luz Elena Mateo-Cid ${ }^{2}$, Á. Catalina \\ Mendoza-González ${ }^{2}$ y Deisy Y. García-López ${ }^{2}$
}

\begin{abstract}
${ }^{1}$ Maestría en Biociencias, Instituto Politécnico Nacional, Escuela Nacional de Ciencias Biológicas, Departamento de Botánica, Carpio y Plan de Ayala Col. Sto. Tomás, México CDMX. 11340, México. *rnavao15@gmail.com

${ }^{2}$ Instituto Politécnico Nacional, Escuela Nacional de Ciencias Biológicas, Departamento de Botánica, Carpio y Plan de Ayala, Col. Sto. Tomás, México CDMX. 11340, México

Abstract.- The seagrass Thalassia testudinum harbors a high specific richness of epiphytic algae due to its morphology and abundance on the coast of the Mexican Atlantic. Epiphytic algae are an important component because of its taxonomic composition and functional groups; additionally, they can be employed as bioindicators. The composition of epiphytic algae of a common forophyte was compared in two ecoregions: Santa Rosa, Quintana Roo with oligotrophic waters and Penacho del Indio, Veracruz with eutrophic waters. Larger coverage of foliate and filamentous algae was expected in the Penacho del Indio prairie due to its eutrophic nature. Seventy seven species of epiphytes were determined, 43 in Penacho del Indio and 46 in Santa Rosa. Overall, 17 species belonged to Cyanobacteria, 38 to Rhodophyta, 11 to Phaeophyceae and 11 to Chlorophyta. Chondria pygmaea, Myrionema magnusii, Hecatonema floridanum, Rosenvingea sanctae-crucis and 7 cyanobacteria represent new registers for the Atlantic coast of Mexico. Centroceras hyalacanthum and Myrionema strangulans are new records for Veracruz. The functional groups were represented by: 39 filamentous algae, 20 microalgae, 9 corticated macrophytes, 5 foliose algae, 3 crustose and one calcareous articulated macrophyte. Red algae displayed the highest specific richness at both sites, followed by green algae in Penacho del Indio and microalgae in Santa Rosa. Epiphytism of foliate, filamentous, as well as corticated macrophytes, had larger coverage and biomass in Penacho del Indio. The prominent development of Ulva at this locality supports its eutrophic condition, whereas epiphytes composition in Santa Rosa suggests the existence of oligotrophic waters.
\end{abstract}

Key words: Epiphytic algae, Caribbean, Gulf of Mexico, seagrasses, Thalassia testudinum

\begin{abstract}
Resumen.- El pasto marino Thalassia testudinum, alberga una alta riqueza específica de epífitas debido a su morfología y abundancia en la costa del Atlántico mexicano. Los epífitos son un importante elemento por su composición taxonómica y grupos funcionales, además pueden utilizarse como bioindicadores. Se comparó la composición de algas epífitas de un forofito común en dos ecorregiones: Santa Rosa, Quintana Roo de aguas oligotróficas y Penacho del Indio, Veracruz de aguas eutróficas. Se esperaba obtener una mayor cobertura de algas foliosas y filamentosas en la pradera de Penacho del Indio debido a su naturaleza eutrófica. Se determinaron 77 especies epífitas, 43 en Penacho del Indio y 46 en Santa Rosa. De ellas, 17 especies corresponden a Cyanobacteria, 38 Rhodophyta, 11 Phaeophyceae y 11 Chlorophyta. Chondria pygmaea, Myrionema magnusii, Hecatonema floridanum, Rosenvingea sanctae-crucis y 7 cianobacterias representan nuevos registros para la costa Atlántica de México. Centroceras hyalacanthum y Myrionema strangulans son nuevos registros para Veracruz. Los grupos funcionales fueron representados por 39 filamentosas, 20 microalgas, 9 macrófitas corticadas, 5 foliosas, 3 costrosas y 1 macrófita articulada calcárea. Las algas rojas presentan una mayor riqueza específica en ambos sitios, seguido por las algas verdes en Penacho del Indio y microalgas en Santa Rosa. El epifitismo de foliosas, filamentosas, así como macrófitas corticadas, tuvo mayor cobertura y biomasa en Penacho del Indio. El notorio desarrollo de Ulva en este sitio corrobora su condición eutrófica, mientras que en Santa Rosa, la composición de epífitas sugiere la presencia de aguas oligotróficas.
\end{abstract}

Palabras clave: Algas epífitas, Caribe, Golfo de México, pastos marinos, Thalassia testudinum 


\section{INTRODUCCIÓN}

Las praderas del pasto marino Thalassia testudinum K.D. König representan un ecosistema costero de gran valor en la biósfera, principalmente por los servicios ecosistémicos que ofrecen, tal como el amortiguamiento de la energía del oleaje, retención de sedimentos y protección contra la erosión (Dawes 1987, Hemminga \& Duarte 2000, Creed et al. 2003, Terrados \& Borum 2004, Brown 2005, Martínez-Daranas 2007).

Estos ambientes costeros generan una alta productividad primaria y diversidad biológica, ofrecen refugio y zonas de crianza en etapas juveniles de especies de importancia comercial como la langosta espinosa, algunos peces y camarones (Terrados \& Borum 2004, Lara-Domínguez 2005, Albis-Salas 2010, López-Calderón \& Riosmena-Rodríguez 2010, van Tussenbroek et al. 2010), además las hojas de la fanerógama y epífitos forman parte de la dieta de especies en conservación como tortugas y de forma indirecta de algunas aves acuáticas (Spalding et al. 2003, López-Calderón \& Riosmena-Rodríguez 2010).

La estructura vertical y horizontal de las plantas de pasto marino propicia la aparición de una gran cantidad de microhábitats, transformando a las hojas en un sustrato ideal para el desarrollo de una alta carga de organismos epífitos (Humm 1964, Díaz-Merlano et al. 2003, Barrios \& Díaz 2005, Lara-Domínguez 2005).

El epifitismo es definido como una forma de vida que ocurre cuando un organismo crece sobre otro organismo vegetal, el que adquiere el nombre de forofito (Borowitzka \& Lethbridge 1989, Ceja-Romero et al. 2008). Esta relación biótica es considerada una posible estrategia ecológica, ya que el epífito obtiene un sustrato en óptimas condiciones de luz y protección, favoreciendo su establecimiento y desarrollo (Mateo-Cid $e t$ al. 2014). Por otra parte, el pasto marino encuentra en las epífitas una protección contra la excesiva radiación UV y ante la desecación durante periodos de baja marea, cuando se encuentra expuesto directamente al aire (van Montfrans et al. 1984, Littler \& Littler 1999). La composición de las algas epífitas depende de su ciclo de vida, biología reproductiva y la capacidad de dispersión (Albis-Salas 2010), principalmente por el tiempo de vida de las hojas de Thalassia Banks ex König, que se encuentra entre los 30 a 60 días (Zieman 1982).

El estudio del epifitismo es una variable importante a considerar en los ambientes marinos, ya que incrementa el conocimiento sobre la riqueza específica de algas marinas en cada zona en estudio y puede ser utilizado para interpretar la estructura de las comunidades marinas bentónicas (Ballantine 1979, Montañés et al. 2003, Ortuño-Aguirre \& RiosmenaRodríguez 2007, Széchy \& Sá 2008).
Asimismo, se ha demostrado que los epífitos pueden ser buenos indicadores de las condiciones del ambiente, si se analiza su composición por especies, abundancia y la presencia de grupos funcionales (Alfonso \& Martínez-Daranas 2009, AlbisSalas 2010). Por ejemplo, se ha descrito el aumento en la concentración de nutrientes inorgánicos relacionados a la abundancia de epífitas con ciclos de vida cortos en aguas someras de zonas costeras (Sand-Jensen \& Borum 1991, Duarte 1995, Valiela et al. 1997). También hay estudios que relacionan los grupos funcionales con variables ambientales, como los de Alfonso \& Martínez-Daranas (2009) para el macrofitobentos y Ávila-Alonso et al. (2013) para las macroalgas asociadas a pastos marinos.

México posee grandes extensiones de praderas de pastos marinos a lo largo del litoral de la costa Atlántica, Caribe, Golfo de California, litoral de Baja California y Baja California Sur, excepto en el Pacífico tropical mexicano (Lara-Domínguez 2005). En las costas de Baja California habitan cuatro especies de pastos marinos, siendo la dominante Zostera marina Linnaeus (López-Calderón \& Riosmena-Rodríguez 2010, López-Calderón et al. 2013). Para el Golfo de México y Caribe, se ubican 5 especies desde Tamaulipas hasta el sistema arrecifal de Yucatán (Lara-Domínguez 2005), donde la especie Thalassia testudinum es la más común y abundante (den Hartog 1970, Díaz-Merlano et al.2003).

El estudio de los epífitos en las costas mexicanas comienza con Ibarra-Obando \& Aguilar-Rosas (1985) y Sánchez-Lizaso \& Riosmena-Rodríguez (1997), quienes trabajaron con Zostera marina y algunas macroalgas marinas. Continuaron MateoCid et al. (2006), estudiando diversos forofitos, Ortuño-Aguirre \& Riosmena-Rodríguez (2007) analizando los epífitos de Padina concrescens Thivy, y Mateo-Cid et al. (2014) con Sargassum sinicola Setchell \& N.L.Gardner.

En cuanto a la composición específica, en Isla Verde, MateoCid et al. (1996) obtuvieron 12 especies de epífitas exclusivas de T. testudinum, de las cuales Hydrolithon farinosum (J.V.Lamouroux) Penrose \& Y.M.Chamberlain y Pneophyllum fragile Kützing se ubicaron como dominantes en las hojas del pasto. Posteriormente, Sepúlveda-Lozada (2004) menciona la dominancia de diatomeas y de cianobacterias en hojas de $T$. testudinum de una zona arrecifal. Okolodkov et al. (2012) muestran información sobre el microfitobentos, incluidas las algas epífitas de T. testudinum. Robinson et al. (2012) presentan nuevos registros de epífitas de T. testudinum, dos algas pardas: Ectocarpus rallsiae Vickers y Cladosiphon occidentalis Kylin. 
Respecto a los estudios para el Caribe mexicano, se presentan los listados florísticos realizados por Huerta-Múzquiz et al. (1987), quienes encontraron 133 epífitas para la Península de Yucatán. En Quintana Roo, Mateo-Cid \& Mendoza-González (1991) determinaron 114 epífitas en Isla Cozumel, además Mendoza-González \& Mateo-Cid (1992) registraron 59 especies para Isla Mujeres y Quan-Young et al. (2006) encontraron 96 epífitas en Bajo Pepito, Isla Mujeres.

De acuerdo a los párrafos anteriores se desprende que el conocimiento de las algas epífitas en T. testudinum se encuentra en publicaciones puntuales. Por lo tanto, se requiere de una ampliación de los estudios de las algas epífitas de este forofito, centrándose en sitos donde no se han documentado epífitas con anterioridad, tal es el caso de la playa Santa Rosa, al sur de Quintana Roo y Penacho del Indio en Veracruz.

La playa Penacho del Indio se encuentra en la ecorregión del Golfo de México sur, caracterizada por aguas costeras eutróficas (Wilkinson et al. 2009) y la playa Santa Rosa en la ecorregión del mar Caribe, constituida por aguas tropicales someras pobres en nutrientes (Wilkinson et al. 2009).

En este contexto, el objetivo del presente trabajo fue comparar el epifitismo del pasto marino T. testudinum entre playa Santa Rosa y Penacho del Indio, a través de un análisis de la riqueza específica y composición de grupos funcionales de macroalgas, microalgas y cianobacterias. Esto permite elaborar un estudio base, para evaluar el impacto de las alteraciones antropogénicas (e.g., turismo) y naturales (e.g., huracanes y arribazones algales), que afectan a las praderas de T. testudinum. Se esperaba que la cobertura de algas foliosas y filamentosas fuera mayor en la pradera localizada en Penacho del Indio, debido a su naturaleza eutrófica.

\section{Materiales y MÉTODOS}

El presente estudio se realizó en dos poblaciones de $T$. testudinum, una de ellas ubicada en el estado de Veracruz, playa Penacho del Indio (1909'04'N N 9605'34”'W) y la otra en el litoral sur del estado de Quintana Roo, playa Santa Rosa (1830’31”N; 8745’32”W), México (Fig. 1).

La playa Penacho del Indio está ubicada $2 \mathrm{~km}$ al norte de Boca del Río y 6 km al norte del Río Jamapa, en la cercanía del arrecife Ingenieros. De acuerdo a la clasificación de Köppen modificada por García (1973), se trata de un clima Aw2, cálido subhúmedo, temperatura media anual mayor de $22^{\circ} \mathrm{C}$ y temperatura del mes más frío mayor de $18^{\circ} \mathrm{C}$ y la precipitación del mes más seco entre 0 y 60 mm (García 1973). Presenta para abril una temperatura media de $25,2^{\circ} \mathrm{C}$. La precipitación promedio es de 50,4 mm (Sistema Meteorológico Nacional 2017). Esta pradera se encuentra dominada por el pasto marino Thalassia testudinum, asociado a macroalgas como Ulva Linnaeus y Gracilaria Greville, además de encontrarse bajo el impacto antropogénico causado por el turismo (descargas hoteleras, restaurantes y diversas actividades recreativas) (Merino 1987).

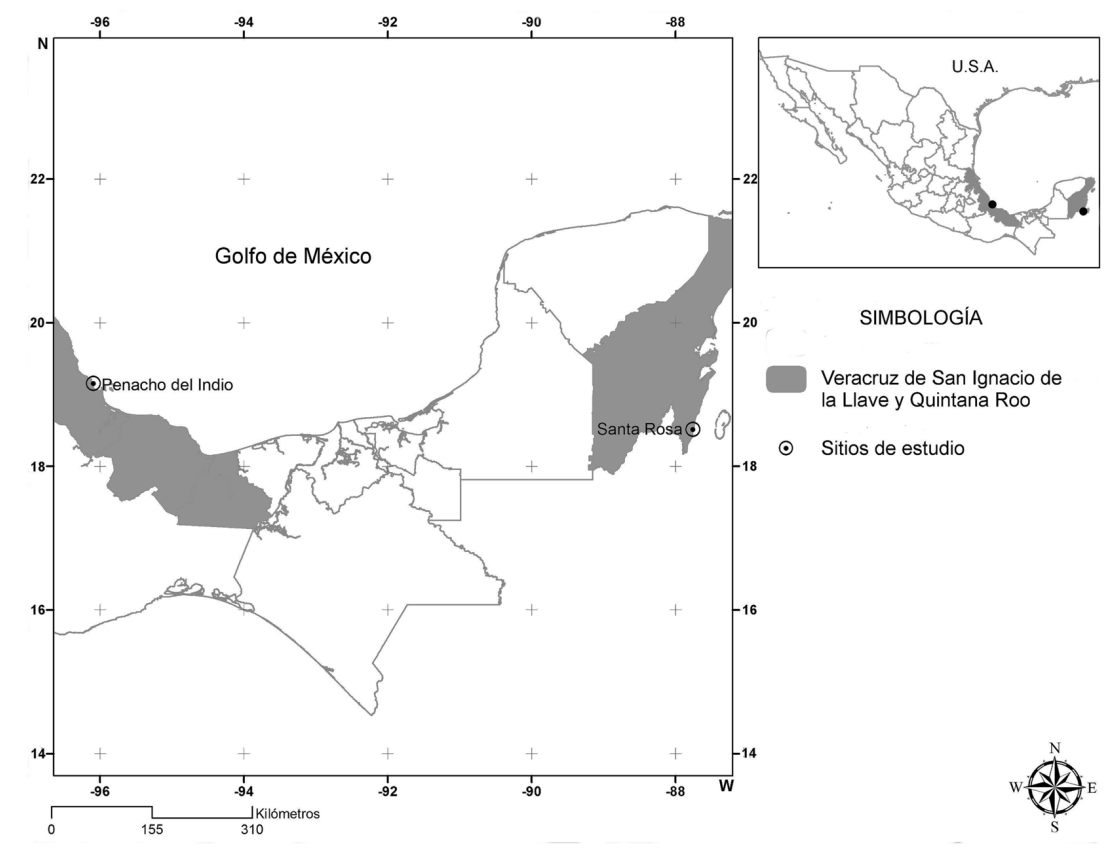

Figura 1. Sitios de estudio. Penacho del Indio en Veracruz y Santa Rosa en Quintana Roo, México / Study sites. Penacho del Indio in Veracruz and Santa Rosa in Quintana Roo, Mexico 
La playa de Santa Rosa, ubicada a $1 \mathrm{~km}$ al norte del Parque Nacional Arrecifes de Xcalak y $23 \mathrm{~km}$ al sur del complejo turístico Mahahual, se trata de un clima Aw2 (x'), cálido subhúmedo, temperatura media anual mayor de $22^{\circ} \mathrm{C}$ y la temperatura de mes más frío mayor de $18^{\circ} \mathrm{C}$. La precipitación del mes más seco está entre 0 y 60 mm (García 1973). En abril se registra el promedio más bajo del año de precipitaciones $\left(18,4 \mathrm{~mm}\right.$ ) y la temperatura media es de $29,5^{\circ} \mathrm{C}$ (Sistema Meteorológico Nacional 2017). Se caracteriza por la ausencia de descargas de agua de ríos debido a la filtración del agua de lluvia a través de la roca caliza (Arriaga-Cabrera et al. 2000). Esta pradera se encuentra dominada por Thalassia testudinum, asociada a la planta marina Syringodium filiforme Kützing y macroalgas como Halimeda J.V.Lamouroux, Penicillus Lamarck, Udotea J.V.Lamouroux y Avrainvillea Decaisne. En las cercanías se encuentran asentamientos humanos dedicados a la pesca artesanal.

Se realizó un muestreo en abril de 2015, en la temporada climática de secas. Se establecieron 3 transectos de $25 \mathrm{~m}$ a lo largo de cada pradera, posicionados de forma perpendicular a la línea de costa y a 25 m entre sí. Se utilizaron 5 unidades de muestreo de $0,0625 \mathrm{~m}^{2}$ (cuadrante) (Ierodiaconou \& Laurenson 2002), los que se colocaron a lo largo de la línea del transecto cada $5 \mathrm{~m}$ ( $\mathrm{n}=15$ por cada pradera). Se recolectaron todos los vástagos de $T$. testudinum (forofito) de las 15 unidades de muestreo mediante buceo autónomo, el material biológico fue depositado en bolsas de plástico.

Simultáneamente, se registró profundidad del sitio mediante un estadal con cinta métrica. Las profundidades de estudio estuvieron entre 0,06-1,4 m en Santa Rosa y entre 0,5-0,8 m en Penacho del Indio. Las muestras se fijaron con formalina al $4 \%$ en agua de mar.

En el laboratorio, las macroalgas epífitas se retiraron de las hojas de la fanerógama. Los representantes de la familia Corallinaceae se trataron con $\mathrm{HNO}_{3}$ 0,6 M para su descalcificación. En el caso de las microalgas y cianobacterias se obtuvieron mediante raspados de las hojas. Para la observación de todos los epífitos se empleó un microscopio estereoscópico Zeigen y un microscopio binocular óptico de campo claro Olympus CX31RBSF (4x, 10x, 40x y 100x).

La determinación de especies se llevó a cabo mediante los trabajos de Taylor (1960), Abbott \& Hollenberg (1976), van den Hoek (1982), Anagnostidis \& Komárek (1988), Schneider \& Searles (1991), Irvine \& Chamberlain (1994), Littler \& Littler (2000), Guimarães et al. (2004), Cho et al. (2008), Won et al. (2009). La secuencia del listado taxonómico siguió el orden propuesto por Anagnostidis \& Komárek (1988) para Cyanobacteria y el de Wynne (2011) para Rhodophyta, Phaeophyceae y Chlorophyta. Las actualizaciones nomenclaturales se obtuvieron de la base de datos taxonómicos
Algaebase (Guiry \& Guiry 2017). Para caracterizar a los grupos funcionales se utilizó la clasificación de Steneck \& Dethier (1994). Asimismo, para determinar la abundancia-dominancia de la superficie epífitada se usó la escala de Braun-Blanquet (Braun-Blanquet \& Pavillard 1922, Braun-Blanquet 1959, 1964) utilizada por Boudouresque (1971) para el fitobentos. Se eligieron 5 vástagos de forma aleatoria por unidad de muestreo y mediante un microscopio estereoscópico se determinó el área cubierta de las epífitas en los primeros $10 \mathrm{~cm}$ de la zona apical de ambos lados de la hoja, empleando placas de Petri cuadriculadas. Se usó el índice de Jaccard para expresar el grado en el que dos muestras son semejantes por las especies de algas epífitas presentes en ellas, por lo que son una medida inversa de la diversidad (Pielou 1975, Magurran 1988).

\section{Resultados}

Durante el estudio completo se determinaron 77 especies (Tabla 1), de las cuales 43 se ubicaron en Penacho del Indio y 46 en Santa Rosa. Del total de ellas, 17 pertenecen a Cyanobacteria, 38 Rhodophyta, 11 Phaeophyceae y 11 Chlorophyta (Fig. 2). Chondria pygmaea Garbary \& Vandermeulen, Myrionema magnusii (Sauvageau) Loiseaux, Hecatonema floridanum (W.R.Taylor) W.R.Taylor y Rosenvingea sanctae-crucis Børgesen representan nuevos registros para la costa Atlántica de México. Mientras que Centroceras hyalacanthum Kützing y Myrionema strangulans Greville son nuevos registros para el litoral de Veracruz. De las 17 especies de cianobacterias registradas, 7 son nuevos registros para el Atlántico mexicano (Tabla 1).

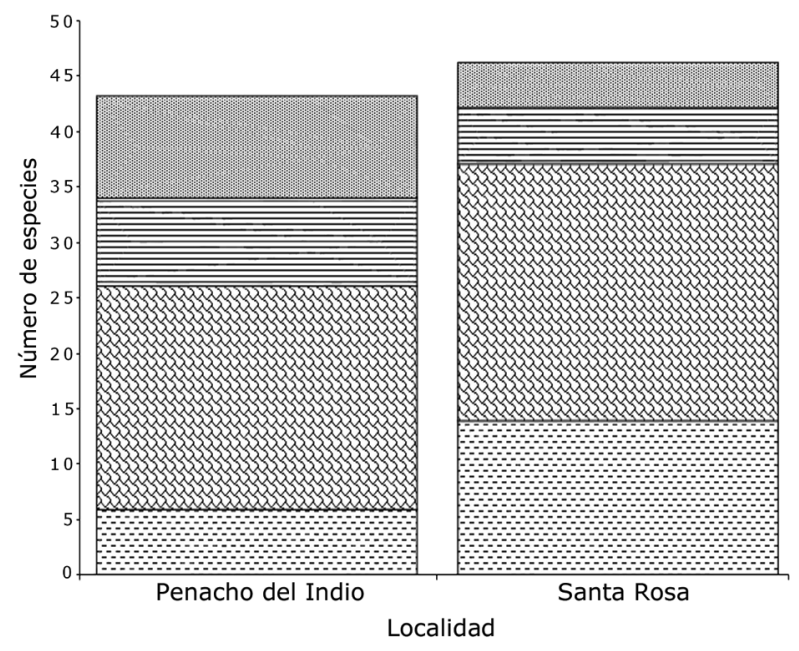

Grupo taxonómico

国 Chlorophyta 目 Phaeophyceae Rhodophyta 긍 Cyanobacteria

Figura 2. Número de especies determinadas por taxón en los dos sitios de estudio / Number of species per taxon at each study site 
Tabla 1. Lista de taxa infragenéricos encontrados en los sitios de estudio, indicando su presencia, abundancia-dominancia. GFM (grupo funcional) y estado reproductivo/ List of the infrageneric taxa found in the study sites, indicating their presence, abundance-dominance. GFM (functional groups) and reproductive status

\begin{tabular}{|c|c|c|c|c|c|c|c|c|c|c|c|}
\hline \multirow[b]{2}{*}{ Taxa } & \multicolumn{2}{|c|}{ Localidad } & \multirow[b]{2}{*}{ GFM } & \multirow[b]{2}{*}{ REPR. } & \multirow[b]{2}{*}{ OBS. } & \multirow[b]{2}{*}{ Taxa } & \multicolumn{2}{|c|}{ Localidad } & \multirow[b]{2}{*}{ GFM } & & \\
\hline & $\begin{array}{l}\text { Santa } \\
\text { Rosa }\end{array}$ & $\begin{array}{l}\text { Penacho } \\
\text { del Indio }\end{array}$ & & & & & $\begin{array}{l}\text { Santa } \\
\text { Rosa }\end{array}$ & $\begin{array}{l}\text { Penacho } \\
\text { del Indio }\end{array}$ & & REPR. & OBS. \\
\hline CYANOBACTERIA & & & & & & 37. Gayliella mazoyerae T.O. Cho, Fredericq \& & $1 / 1$ & $1 / 3$ & II & $\operatorname{Tr}, \mathrm{Ci}, \mathrm{d}$ & \\
\hline Aphanothecaceae & & & & & & Hommersand & & & & & \\
\hline $\begin{array}{l}\text { 1. Aphanothece granulosa (N.L.Gardner) Komárek \& } \\
\text { Komákova-Legnerová }\end{array}$ & $1 /+$ & 0 & I & $\mathrm{Ve}$ & NAM & $\begin{array}{l}\text { Wrangeliaceae } \\
\text { 38. Anotrichium cf. secundum (Harvey ex J. Agardh) }\end{array}$ & & & & & \\
\hline Entophysalidaceae & & & & & & G. Furnari & $1 / 1$ & 0 & II & $\operatorname{Tr}$ & \\
\hline 2. Entophysalis conferta (Kützing) Drouet \& Daily & $1 /+$ & $1 /+$ & I & Ve & & Rhodomelaceae & & & & & \\
\hline Coleofasciculaceae & & & & & & 39. Chondria baileyana (Montagne) Harvey & 0 & $1 / 3$ & IV & $\operatorname{Tr}$ & \\
\hline 3. Coleofasciculus chthonoplastes (Thuret ex Gomont) & $1 /+$ & $1 /+$ & I & $\mathrm{Hm}$ & & 40. Chondria collinsiana M. Howe & 0 & $1 / 3$ & IV & $\mathrm{Tr}, \overline{0}, \mathrm{Ci}$ & \\
\hline M.Siegesmund, J.R. Johansen \& T. Friedl & & & & & & 41. Chondria curvilineata Collins \& Hervey & $1 / 1$ & 0 & IV & $\operatorname{Tr}$ & \\
\hline $\begin{array}{l}\text { Heteroleibleiniaceae } \\
\end{array}$ & & & & & & 42. Chondria leptacremon (Melvill ex G. Murray) De & 0 & $1 / 3$ & IV & $\mathrm{Tr}$ & \\
\hline 4. Heteroleibleinia infixa (Frémy) Anagnostidis \& & 0 & $1 /+$ & I & $\mathrm{Ve}$ & & Toni & & & & & \\
\hline Komárek & & & & & & 43. Chondria pygmaea Garbary \& Vandermeulen & $1 / 2$ & 0 & IV & $\operatorname{Tr}$ & NM \\
\hline Leptolyngbyaceae & & & & & & 44. Herposiphonia secunda (C. Agardh) Ambron & $1 / 1$ & 0 & II & $\mathrm{Ci}, \mathrm{Tr}, \hat{\partial}$ & \\
\hline 5. Trichocoleus polythrix (Forti) Anagnostidis & $1 / 4$ & 0 & I & Ve & NAM & 45. Laurencia caduciramulosa Masuda \& S. & $1 / 2$ & 0 & IV & $\mathrm{Ve}$ & \\
\hline Cyanothecaceae & & & & & & Kawaguchi & & & & & \\
\hline 6. Cyanothece halobia Roussomoustakaki \& & $1 /+$ & 0 & I & & NAM & 46. Lophosiphonia cristata Falkenberg & $1 / 1$ & 0 & II & $\mathrm{Ci}, \mathrm{Tr}, 0 \overrightarrow{\mathrm{O}}$ & \\
\hline Anagnostidis & & & & & & 47. Lophosiphonia obscura (C. Agardh) Falkenberg & $1 / 1$ & 0 & II & $\mathrm{Ci}, \mathrm{Tr}, \partial^{2}$ & \\
\hline Microcolaceae & & & & & & 48. Polysiphonia atlantica Kapraun \& J.N. Norris & 0 & $1 / 4$ & II & $\hat{0}$ & \\
\hline 7. Microcoleus autumnalis (Gomont) Strunecky, & 0 & $1 /+$ & I & & & 49. Polysiphonia binneyi Harvey & $1 / 1$ & $1 / 4$ & II & $\operatorname{Tr}, \widehat{\partial}$ & \\
\hline Komárek \& J.R. Johansen & & & & & & 50. Polysiphonia scopulorum Harvey & $1 / 1$ & 0 & II & $\mathrm{Tr}, \overline{\mathrm{O}}, \mathrm{Ci}$ & \\
\hline Oscillatoriaceae & & & & & & 51. Melanothammus ferulaceus (Suhr ex J. Agardh) & 0 & $1 / 4$ & II & $\mathrm{Pl}$ & \\
\hline 8. Lyngbya aestuarii Liebman ex Gomont & $1 /+$ & 0 & I & & & Díaz-Tapia \& Maggs & & & & & \\
\hline 9. Oscillatoria funiformis (Vouk) Komárek & $1 /+$ & 0 & I & & & 52. Melanothammus gorgoniae (Harvey) Díaz-Tapia & $1 / 1$ & $1 / 4$ & II & $\operatorname{Tr}$ & \\
\hline Spirulinaceae & & & & & & \& Maggs & & & & & \\
\hline 10. Spirulina meneghiniana Zanardini ex Gomont & $1 /+$ & 0 & I & & & 53. Melanothammus sphaerocarpus (Børgesen) Díaz- & 0 & $1 / 4$ & II & $\operatorname{Tr}$ & \\
\hline 11. Spirulina robusta H. Welsh & $1 /+$ & 0 & I & & & Tapia \& Maggs & & & & & \\
\hline Scytonemataceae & & & & & & Cystocloniaceae & & & & & \\
\hline 12. Scytonematopsis crustacea (Thuret ex Bornet \& & & & & & & 54. Hypnea spinella $($ C.Agardh) Kützing & 0 & $1 / 2$ & IV & $\mathrm{Ve}$ & \\
\hline Flahault) Koválik \& Komárek & 0 & $1 /+$ & I & $\mathrm{Ht}$ & & Champiaceae & & & & & \\
\hline Rivulariaceae & & & & & & 55. Champia parvula (C.Agardh) Harvey & 0 & $1 / 4$ & IV & $\operatorname{Tr}$ & \\
\hline 13. Calothrix confervicola C.Agardh ex Bornet \& & & & & & & OCHROPHYTA (Phaeophyceae) & & & & & \\
\hline Flahault & $1 /+$ & $1 /+$ & I & $\mathrm{Ht}$ & & Acinetosporaceae & & & & & \\
\hline 14. Calothrix contarenii Bornet \& Flahault & $1 /+$ & 0 & I & $\mathrm{Ht}$ & NAM & 56. Feldmannia irregularis (Kützing) Hamel & 0 & $1 / 1$ & II & $\mathrm{Pl}$ & \\
\hline 15. Dichothrix gypsophila Bornet \& Flahault & $1 /+$ & 0 & I & $\mathrm{Ht}$ & NAM & 57. Feldmannia mitchelliae (Harvey) H.S. Kim & $1 / 4$ & 0 & II & $\mathrm{Pl}$ & \\
\hline 16. Dichothrix ramenskii Elenkin & $1 /+$ & 0 & I & $\mathrm{Ht}$ & NAM & Ectocarpaceae & & & & & \\
\hline 17. Rivularia bornetiana Setchell & $1 /+$ & 0 & I & $\mathrm{Ht}$ & NAM & 58. Ectocarpus rallsiae Vickers & 0 & $1 / 1$ & II & $\mathrm{Pl}$ & \\
\hline RHODOPHYTA & & & & & & 59. Ectocarpus siliculosus (Dillwyn) Lyngbye & 0 & $1 / 1$ & II & $\mathrm{Pl}$ & \\
\hline Stylonemataceae & & & & & & Chordariaceae & & & & & \\
\hline 18. Chroodactylon ornatum (C. Agardh) Basson & $1 /+$ & 0 & II & $\mathrm{Ve}$ & & 60. Cladosiphon zosterae (J.Agardh) Kylin & $1 / 2$ & $1 / 2$ & II & Un & \\
\hline 19. Stylonema alsidii (Zanardini) K.M. Drew & 0 & $1 /+$ & II & $\mathrm{Ve}$ & & 61. Hecatonema floridanum (W.R. Taylor) W.R. & $1 / 1$ & $1 / 1$ & II & Plu & NM \\
\hline Erythrotrichiaceae & & & & & & Taylor & & & & & \\
\hline 20. Erythrotrichia carnea (Dillwyn) J. Agardh & $1 /+$ & 1/+ & II & $\mathrm{Ve}$ & & 62. Myrionema strangulans Greville & 0 & 1 & II & Plu & NV \\
\hline Acrochaetiaceae & & & & & & Bachelotiaceae & & & & & \\
\hline 21. Acrochaetium hoytii Collins & $1 / 1$ & 0 & II & $\mathrm{Mn}$ & & 63. Bachelotia antillarum (Grunow) Gerloff & $1 / 3$ & 0 & II & Plu & \\
\hline 22. Acrochaetium robustum Børgesen & $1 / 1$ & 0 & II & $\mathrm{Mn}$ & & Scytosiphonaceae & & & & & \\
\hline Colaconemataceae & & & & & & 64. Rosenvingea sanctae-crucis Børgesen & 0 & $1 / 2$ & IV & Un & NAM \\
\hline $\begin{array}{l}\text { 23. Colaconema dasyae (Collins) Stegenga, I. Mol, } \\
\text { Prud'homme van Reine \& Lokhorst }\end{array}$ & $1 / 1$ & 0 & II & $\mathrm{Mn}$ & & $\begin{array}{l}\text { Dictyotaceae } \\
\text { 65. Canistrocarpus cervicornis (Kützing) De Paula \& }\end{array}$ & & & & & \\
\hline 24. Colaconema daviesii (Dillwyn) Stegenga & 0 & $1 / 4$ & II & $\mathrm{Mn}$ & & De Clerck & 0 & $1 / 2$ & III & Og & \\
\hline 25. Colaconema hallandicum (Kylin) Afonso- & $1 / 1$ & $1 / 4$ & II & $\mathrm{Mn}$ & & Sphacelariaceae & & & & & \\
\hline Carillo, Sanson, Sangil \& Diaz-Villa & & & & & & 66. Sphacelaria rigidula Kützing & $1 / 1$ & 0 & II & $\mathrm{Pp}$ & \\
\hline 26. Colaconema savianum (Meneghini) R. Nielsen & $1 / 1$ & 0 & II & $\mathrm{Mn}$ & & CHLOROPHYTA & & & & & \\
\hline Hapalidiaceae & & & & & & Phaeophilaceae & & & & & \\
\hline 27. Melobesia membranacea (Esper) J.V. Lamouroux & 0 & 1 & VI & $\operatorname{Tr}$ & & 67. Phaeophila dendroides (P. Crouan \& H. Crouan) & $1 /+$ & $1 /+$ & I & $\mathrm{Ve}$ & \\
\hline Corallinaceae & & & & & & Batters & & & & & \\
\hline 28. Hydrolithon farinosum (J.V. Lamouroux) & & & & & & Ulvaceae & & & & & \\
\hline D. Penrose \& Y.M. Chamberlain & $1 / 4$ & 1 & VI & $\operatorname{Tr}$ & & 68. Ulva compressa Linnaeus & 0 & $1 / 5$ & III & Ve & \\
\hline 29. Jania capillacea Harvey & $1 / 1$ & 0 & $\mathrm{~V}$ & $\mathrm{Ve}$ & & 69. Ulva flexuosa subsp. paradoxa (C. Agardh) M.J. & 0 & $1 / 5$ & III & $\mathrm{Ve}$ & \\
\hline 30. Pneophyllum confervicola (Kützing) Y.M. & $1 / 4$ & 0 & VI & $\operatorname{Tr}$ & & Wynne & & & & & \\
\hline Chamberlain & & & & & & 70. Ulva intestinalis Linnaeus & 0 & $1 / 5$ & III & $\mathrm{Ve}$ & \\
\hline Ceramiaceae & & & & & & 71. Ulva rigida C. Agardh & 0 & $1 / 5$ & III & $\mathrm{Ve}$ & \\
\hline 31. Centroceras gasparrinii (Meneghini) Kützing & 0 & $1 / 3$ & II & 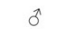 & & Ulvellaceae & & & & & \\
\hline 32. Centroceras hyalacanthum Kützing & 0 & $1 / 3$ & II & $\sigma^{2}$ & NV & 72. Ulvella lens P. Crouan \& H. Crouan & $1 /+$ & $1 /+$ & I & $\mathrm{Ve}$ & \\
\hline 33. Ceramium cruciatum Collins \& Hervey & $1 / 1$ & 0 & II & $\operatorname{Tr}$ & & 73. Ulvella viridis (Reinke) R. Nielsen, C.J. O'Kelly & $1 /+$ & 0 & I & $\mathrm{Ve}$ & \\
\hline 34. Crouania attenuata (C.Agardh) J. Agardh & $1 / 1$ & 0 & II & $\operatorname{Tr}$ & & \& B. Wysor & & & & & \\
\hline 35. Crouanophycus latiaxis (I.A. Abbott) A. & $1 / 1$ & 0 & II & $\operatorname{Tr}$ & & Cladophoraceae & & & & & \\
\hline Athanasiadis & & & & & & 74. Chaetomorpha minima Collins \& Hervey & 0 & $1 / 3$ & II & $\mathrm{Ve}$ & \\
\hline 36. Gayliella flaccida (Harvey ex Kützing) T.O. Cho & 0 & $1 / 3$ & II & 0 & & 75. Cladophora hutchinsiae (Dillwyn) Kützing & 0 & $1 / 3$ & II & $\mathrm{Ve}$ & \\
\hline \& L.J. Mclvor & & & & & & 76. Cladophora liniformis Kützing & $1 / 1$ & 0 & II & $\mathrm{Ve}$ & \\
\hline & & & & & & 77. Cladophora vagabunda (Linnaeus) Hoek & 0 & $1 / 3$ & II & $\mathrm{Ve}$ & \\
\hline
\end{tabular}

$1=$ presencia, $0=$ ausencia; Escala de abundancia-dominancia:,$+ 1=$ menos del $5 \%$ de la superficie, $2=$ entre el 5 y $25 \%$ de la superficie, $3=$ entre el 25 y $50 \%$, $4=$ entre el 50 y $75 \%, 5=$ más del $75 \%$ de la superficie. Grupos funcionales: $\mathrm{I}=$ microalgas, II= algas filamentosas, III= algas foliosas, IV= macrófitas corticadas, V= algas articuladas calcáreas, $\mathrm{VI}=$ algas costrosas. Estructuras reproductoras (REPR.): $\mathrm{Tr}=$ Tetrasporangios, $\mathrm{Pl}=\mathrm{Plurangios}, \mathrm{Ht}=\mathrm{Heterocistos}, \mathrm{Hm}=\mathrm{Hormogonios}, \mathrm{Mn}=$ Monosporas, $\mathrm{Og}=$ Oogonios, $\mathrm{Ci}=$ Carposporangios, $\delta=$ Espermatangios y Ve= Vegetativo. Observaciones (OBS): $\mathrm{NM}=\mathrm{Nuevo}$ registro para México, $\mathrm{NAM}=\mathrm{Nuevo}$ registro para la costa Atlántica de México, NV= Nuevo registro para la costa de Veracruz 
Respecto a los grupos funcionales, se clasificaron en: microalgas (20 especies), algas filamentosas (39 especies), algas foliosas (5 especies), macrófitas corticadas ( 9 especies), macrófitas articuladas calcáreas ( 1 especie) y algas costrosas (3 especies).

En Penacho del Indio, se obtuvieron 5 grupos funcionales: macrófitas corticadas, microalgas, algas filamentosas, costrosas y foliosas. Este último grupo, se encontró sólo en esta población de T. testudinum, con mayor cobertura y biomasa respecto al resto de grupos funcionales, determinado por representantes de los géneros Ulva y Canistrocarpus De Paula \& De Clerck, seguido de macrófitas corticadas de los géneros Chondria C.Agardh, Hypnea J.V.Lamouroux y Champia Desvaux.

En Santa Rosa también se registraron 5 grupos funcionales, pero la composición difirió: algas filamentosas, microalgas, macrófitas corticadas, calcáreas corticadas y costrosas.

Las algas filamentosas fueron las más representadas en ambas localidades por su riqueza específica (Fig. 3, Tabla 1). Al comparar la composición de algas epífitas entre ambas localidades, se obtuvo un valor de similitud de 0,16. Solo 12 especies fueron compartidas, las cuales en su mayoría pertenecían al grupo de filamentosas.

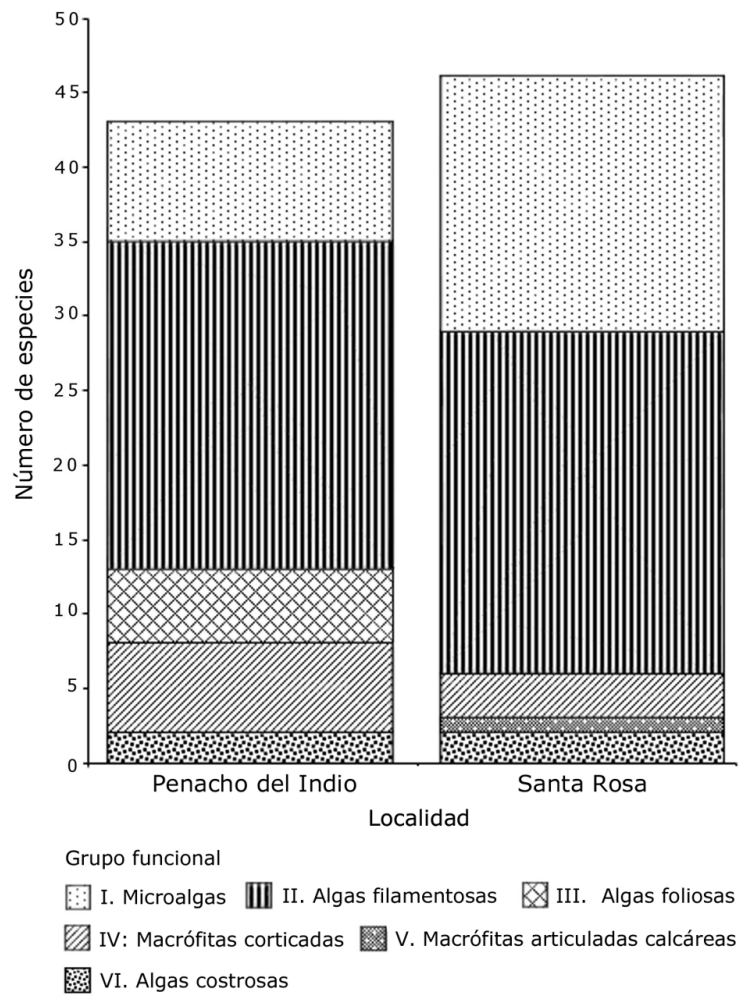

Figura 3. Número de especies determinadas por grupo funcional en los sitios de estudio / Number of species determined by functional group at each study site

\section{Discusión}

En la temporada climática de secas, se encontró un mayor número de especies de algas epífitas en Santa Rosa que las reportadas previamente para el Caribe mexicano por QuanYoung et al. (2006), pero inferior a las registradas por MateoCid \& Mendoza-González (1991) y Mendoza-González \& Mateo-Cid (1992). En el caso de las especies localizadas en Penacho del Indio, Veracruz, se ubicó un número mayor que los determinados por Mateo-Cid et al. (1996) y Robinson et al. (2012), lo que permite fortalecer los registros de riqueza específica para la zona de estudio. Este resultado puede deberse a un mayor esfuerzo de recolecta por unidad de muestreo, así como la revisión minuciosa del forofito que se realizó en el presente estudio para ubicar, separar y determinar las epífitas.

El grupo taxonómico más representado en cuanto a la riqueza específica fue Rhodophyta con 38 especies, lo que resalta su elevada contribución cuantitativa frente al resto de algas. Esta predominancia concuerda con los registros previos en otras regiones, donde se ha documentado que las algas rojas dominan en composición de especies y biomasa (Mendoza-González \& Mateo-Cid 1992, Borowitzka et al. 2006, Quan-Young et al. 2006, Albis-Salas 2010). Asimismo, los registros de estas algas en estudios de floras epífitas, tanto en angiospermas marinas (Ibarra-Obando \& Aguilar-Rosas 1985, Barrios \& Díaz 2005), como en macroalgas (Aguilar-Rosas \& Machado-Galindo 1990, Montañés et al. 2003, Quan-Young et al. 2006, OrtuñoAguirre \& Riosmena-Rodríguez 2007), demuestran que las algas rojas son el grupo con mayor riqueza específica dentro del epifitismo, ya que presentan formas de vida y estrategias reproductivas que les permiten permanecer en el forofito (AlbisSalas 2010).

En cuanto a los grupos funcionales, en Santa Rosa las algas filamentosas fueron las de elevada riqueza específica y las más abundantes respecto a los otros grupos. Estas se ubicaron dispuestas en la zona apical y en los bordes de las hojas de Thalassia, cubriendo en un $90 \%$ su superficie, lo que concuerda con lo observado por otros autores como Heijs (1985), Wear et al. (1999), Ierodiaconou \& Laurenson (2002), Saunders et al. (2003) y Mateo-Cid et al. (2013). Además se encontraron especímenes de los géneros Gayliella T.O.Cho, L.J.McIvor \& S.M.Boo, Herposiphonia Nägeli, Lophosiphonia Falkenberg y Polysiphonia Greville sobre la misma hoja, con 3 diferentes estadios reproductivos: gametangios, carposporangios y tetrasporangios, es decir, las 3 generaciones que constituyen el ciclo de vida de estas algas rojas. El hecho que la fase esporofitica fuera la más abundante representa una ventaja, ya que requieren de un menor gasto energético para la formación de esporas, hay una diseminación en forma rápida, una dispersión eficiente, una alta probabilidad de fijación y 
desarrollo de los talos (Huerta-Múzquiz et al. 1987, MateoCid \& Mendoza-González 1991, Mendoza-González \& Mateo-Cid 1992, Aguilar-Rosas et al. 1998, Mateo-Cid \& Mendoza-González 2007). Es por ello que son consideradas oportunistas y euritípicas, además de encontrarse entre los primeros estadios sucesionales en el forofito (Littler \& Littler 1980).

Cabe resaltar que en la playa Santa Rosa, dentro del grupo de microalgas, se ubicaron 4 especies de cianobacterias de ambientes dulceacuícolas, lo cual puede relacionarse con el aporte de agua dulce que fluye entre la arena por los ríos subterráneos. Además el número de cianobacterias es mayor que en Penacho del Indio. Este grupo es uno de los colonizadores más abundantes en la zona intermareal (Alpuche-Gual 2014). Junto con las diatomeas y bacterias son considerados como los primeros organismos que forman las biopelículas observadas en las hojas más jóvenes en conjunto a las algas coralinas costrosas y algunos invertebrados como hidrozoos y briozoos (Borowitzka et al. 2006). Estas algas coralinas incrustantes son susceptibles a la proliferación de algas filamentosas en zonas someras muy productivas (Sebens 1986, Steneck 1986).

Las algas coralinas Hydrolithon y Pneophyllum fueron encontradas en todas las hojas de Thalassia, desde las más jóvenes hasta las senescentes. Estos organismos una vez asentados y establecidos, pueden persistir durante toda la vida útil del forofito (Borowitzka et al. 2006). Este grupo es considerado como abundante y con menor riqueza específica sobre las hojas de pastos marinos (Leliaert et al. 2001, Barrios \& Díaz 2005, Balata et al. 2007, Mateo-Cid et al. 2013), hecho que fue observado en este estudio. La gran cantidad de esporas encontradas en las muestras se relacionan con su éxito reproductivo, además de su morfología plana incrustante, que resiste con mayor facilidad la tensión física del ambiente (Steneck 1986).

Es conveniente mencionar que los meses de abril a septiembre corresponden a la temporada de mayor reproducción de T. testudinum para México (van Tussenbroek 2010). La gran biomasa y cobertura de epífitas encontradas en el muestreo se relaciona con la inversión energética de Thalassia en su reproducción y no en los mecanismos de defensa contra las epífitas, como se ha descrito en otros forofitos (Bulthuis \& Woelkerling 1983, Short \& Burdick 1996, Sureda et al. 2008).

Los resultados obtenidos en Penacho del Indio indican una alta cobertura y riqueza específica (Tabla 1), como lo menciona Albis-Salas (2010), en relación con aportes de nutrientes derivados del turismo. Respecto a los grupos funcionales de algas foliosas, filamentosas y macrófitas corticadas fueron de mayor cobertura sobre el forofito (Fig. 3), encontradas en cerca del $100 \%$ de la superficie de las hojas y con una elevada biomasa. La abundancia de las algas filamentosas y las foliosas se encuentra relacionado con el aporte de nutrientes (Alfonso \& Martínez-Daranas 2009). Además se observaron densos agregados de algas foliosas asociadas al rizoma de Thalassia, tales como Ulva lactuca Linnaeus y $U$. intestinalis Linnaeus. Estos datos podrían relacionarse con las descargas de residuos hoteleros y del turismo que pueden ser observados en el sitio de muestreo, conjuntamente con la cercanía del río Jamapa. Valores elevados de biomasa (O'Shanahan et al. 2003, Wan et al. 2017) y crecimiento excesivo (Fletcher et al. 1990, Anderson et al. 1996, Calva \& Torres-Alvarado 2008) de algas verdes se han relacionado con condiciones de eutrofización. Más aun, Ho (1987) menciona a una gran biomasa de Ulva como bioindicador del aporte de aguas residuales. En este contexto, la presencia de las algas foliosas representadas por el género Ulva podría estar relacionada con el aporte de nutrientes debido a las descargas de residuos hoteleros y el turismo en la zona de estudio.

Por otra parte, la gran cantidad de macrófitas corticadas encontradas en Penacho del Indio podría relacionarse con valores elevados de oxígeno disuelto como lo mencionan ÁvilaAlonso et al (2013), haciendo necesario la realización de estudios periódicos para relacionar las variables ambientales y los grupos funcionales.

En lo que respecta a la composición de epífitas, sólo el 16\% de ellas se encuentran en ambas regiones, las que en su gran mayoría son algas filamentosas. Estas son especies de amplia distribución, en contraste con las especies exclusivas de cada sitio. La riqueza específica fue semejante entre ambos sitios de estudio.

De esta forma, se comprueba la diferencia en la composición de grupos funcionales, así como en su cobertura y biomasa entre los sitios de estudio. La mayor cobertura y biomasa fue encontrada en las aguas eutróficas de Penacho del Indio, destacando las algas foliosas, filamentosas y macrófitas corticadas. Mientras que en las aguas oligotróficas del Caribe donde se ubica la playa Santa Rosa, sólo destacan las algas filamentosas, aunque con una menor biomasa y cobertura. Se recomienda realizar estudios mensuales y cuantitativos en ambos sitios, que nos permitan determinar la calidad del agua, así como el aporte de nutrientes, los que podrían relacionarse con la composición taxonómica y funcional de epífitas. Por ello, la actual investigación es un primer esfuerzo en utilizar grupos funcionales de algas epífitas y especies indicadoras para caracterizar el hábitat de estudio. 


\section{Agradecimientos}

Al Instituto Politécnico Nacional, Proyectos SIP-20150767, 20150850, 20161052 y 20164772, por facilitar el apoyo económico, las instalaciones y equipo necesario para el desarrollo de este estudio. La primera autora agradece al Consejo Nacional de Ciencia y Tecnología (CONACyT) la beca otorgada. La segunda y tercera autora agradecen la beca otorgada por la Comisión de Operación y Fomento de Actividades Académicas (COFAA) y los incentivos del programa al Estímulo de los Investigadores (EDI), a Xhail Flores por la elaboración de la figura del área de estudio. A Julio Acosta Calderón y Adrián Gerardo A. Garduño Acosta por el apoyo logístico.

\section{LITERATURA CITADA}

Abbott I \& G Hollenberg. 1976. Marine algae of California, 230 pp. Stanford University Press, Stanford.

Aguilar-Rosas MA, LE Aguilar \& R Aguilar. 1998. Algas marinas de la región central de Quintana Roo, México, Polibotánica 7: 15-32.

Aguilar-Rosas R \& R Machado-Galindo. 1990. Ecological aspects of Sargassum muticum (Fucales, Phaeophyta) in Baja California, México: Reproductive phenology and epiphytes. Hydrobiologia 204/205: 185-190.

Albis-Salas MR. 2010. Características estructurales y fisiológicas de las praderas de Thalassia testudinum. Tesis de Maestría, Universidad Nacional de Colombia, Santa Marta, 128 pp.

Alfonso Y \& B Martínez-Daranas. 2009. Variaciones espaciotemporales en la cobertura del macrofitobentos en un área costera al norte de la ciudad de la Habana, Cuba. Revista de Investigaciones Marinas 30(3): 187-201.

Alpuche-Gual L. 2014. Clasificación de playas campechanas para su manejo integral y desarrollo sostenible, $156 \mathrm{pp}$. Universidad Autónoma de Campeche, San Francisco de Campeche.

Anagnostidis K \& J Komárek. 1988. Modern approach to the classification system of Cyanophytes. 3. Oscillatoriales. Algological Studies 50-53: 327-472.

Anderson RJ, PMS Monteiro \& GJ Levi. 1996. The effect of localized eutrophication on competition between Ulva lactuca (Ulvaceae, Chlorophyta) and a commercial resource of Gracilaria verrucosa (Gracilariaceae, Rhodophyta). Hydrobiologia 326-327: 291-296.

Arriaga-Cabrera L, V Aguilar-Sierra \& J Alcocer-Durand. 2000. Aguas continentales y diversidad biológica de México, 325 pp. Comisión Nacional para el Conocimiento y Uso de la Diversidad, Ciudad de México.

Ávila-Alonso D, M Guimarães \& R Cárdenas. 2013. Variación espacio-temporal de grupos morfo-funcionales de macroalgas en pastos marinos al norte de Ciego de Ávila, Cuba. Revista de Investigaciones Marinas 33(1): 14-22.
Balata D, U Nesti, L Piazzi \& F Cinelli. 2007. Patterns of spatial variability of seagrass epiphytes in the north-west Mediterranean Sea. Marine Biology 151: 2025-2035.

Ballantine DL. 1979. The distribution of algal epiphytes on macrophyte hosts offshore from La Parguera, Puerto Rico. Botanica Marina 22: 107-111.

Barrios J \& O Díaz. 2005. Algas epífitas de Thalassia testudinum en el Parque Nacional Mochima, Venezuela. Boletín del Centro de Investigaciones Biológicas 39: 1-14.

Borowitzka M \& R Lethbridge. 1989. Seagrasses epiphytes. In: Larkum AWD, AJ McComb \& SA Shepherd (eds). Biology of seagrasses, pp. 458-499. Elsevier, Amsterdam.

Borowitzka M, P Lavery \& M Keulen. 2006. Epiphytes of seagrasses. In: Larkum AWD, RJ Orth \& CM Duarte (eds). Seagrasses: Biology, ecology and conservation, pp. 441-461. Springer-Verlag, Berlin.

Boudouresque CF. 1971. Méthodes d'étude qualitative et quantitative du benthos (en particulier du phytobenthos). Tethys 3(1): 79-104.

Braun-Blanquet J. 1959. Grundfragen und Aufgaben des Pflanzensoziologie. In: Turril WB (ed). Vistas in botany, pp. 145-171. Pergamon Press, London.

Braun-Blanquet J. 1964. Pflanzensoziologie, 865 pp. Springer Verlag, Wien.

Braun-Blanquet J \& J Pavillard. 1922. Vocabulaire de sociologie végétale, $23 \mathrm{pp}$. Romégou et Dehan, Montpellier.

Brown KM. 2005. Calcareous epiphytes on modern seagrasses as carbonate sediment producers in shallow cool-water marine environments, South Australia. Tesis Doctoral, University of Adelaide, Adelaide, $247 \mathrm{pp}$.

Bulthuis DA \& WJ Woelkerling. 1983. Biomass accumulation and shading effects of epiphytes on leaves of the seagrass Heterozostera tasmanica, in Victoria, Australia. Aquatic Botany 16: 137-148.

Calva L \& R Torres-Alvarado. 2008. Macroalgas y pasto marino, útiles bioindicadores de contaminación por hidrocarburos fósiles en sistemas acuáticos. ContactoS 68: $38-46$.

Ceja-Romero J, A Espejo, AR López, J García, A Mendoza \& B Pérez. 2008. Las plantas epífitas, su diversidad e importancia. Ciencias 91:35-41.

Cho T, S Boo, M Hommersand, C Maggs, L McIvor \& S Fredericq. 2008. Gayliella gen. nov. in the tribe Ceramiaceae (Ceramiaceae, Rhodophyta) based on molecular and morphological evidence. Journal of Phycology 44: 721-738.

Creed JC, RC Phillips \& BI van Tussenbroek. 2003. The seagrasses of the Caribbean. In: Green EP \& FT Short (eds). World atlas of seagrasses, pp. 234-242. UNEP World Conservation Monitoring Centre, University of California Press, Berkeley.

Dawes CJ. 1987. The dynamic seagrasses of the Gulf of Mexico and Florida Coasts. Florida Marine Research Publications 42:25-38. 
den Hartog C. 1970. The sea-grasses of the world, 275 pp. North-Holland Publishing Co., Amsterdam.

Díaz-Merlano JM, L Barrios \& D Gómez-López. 2003. Las praderas de pastos marinos en Colombia: Estructura y distribución de un ecosistema estratégico, 160 pp. INVEMAR, Serie Publicaciones Especiales 10, Santa Marta.

Duarte CM. 1995. Submerged aquatic vegetation in relation to different nutrient regimes. Ophelia 41:87-112.

Fletcher RL, V Cuomo \& I Palomba. 1990. The 'green tide' problem, with particular reference to the Venice Lagoon. British Phycological Journal 25(1): 87.

García E. 1973. Modificaciones del Sistema de Clasificación Climática de Köppen (para adaptarlas a las condiciones de la República Mexicana), 246 pp. Instituto de Geografía, Universidad Nacional Autónoma de México, Ciudad de México.

Guimarães S, M Fujii, D Pupo \& N Yokoya. 2004. Reavaliação das características morfológicas e suas implicações taxonômicas no gênero Polysiphonia sensu lato (Ceramiales, Rhodophyta) do litoral dos Estados de São Paulo e Espírito Santo, Brasil. Revista Brasileira de Botânica 27: 163-183.

Guiry MD \& GM Guiry. 2017. AlgaeBase. World-wide electronic publication, National University of Ireland, Galway <http://www.algaebase.org>

Heijs FML. 1985. The seasonal distribution and community structure of the epiphytic algae on Thalassia hemprichii (Ehrenb.) Aschers from Papua New Guinea. Aquatic Botany 21:295-324

Hemminga M \& C Duarte. 2000. Seagrass ecology, 298 pp. University of Cambridge, New York.

Ho YB. 1987. Ulva lactuca (Chlorophyta, Ulvales) in Hong Kong intertidal waters. Its nitrogen and phosphorus contents and its use as a bioindicator of eutrophication. Asian Marine Biology 4: 97-102.

Huerta-Múzquiz L, AC Mendoza-González \& LE MateoCid. 1987. Avance sobre un estudio de las algas marinas de la Península de Yucatán. Phytologia 62: 22-53.

Humm H. 1964. Epiphytes of the seagrass, Thalassia testudinum, in Florida. Bulletin of Marine Science 14: 306341.

Ibarra-Obando S \& R Aguilar-Rosas. 1985. Macroalgas flotantes y epífitas asociadas con Zostera marina L. en Bahía San Quintín (B.C., México) durante verano-otoño 1982: Biomasa y composición taxonómica. Ciencias Marinas 11: 89-104.

Ierodiaconou DA \& LJB Laurenson. 2002. Estimates of Heterozostera tasmanica, Zostera muelleri and Ruppia megacarpa distribution and biomass in the Hopkins Estuary, Western Victoria, by GIS. Australian Journal of Botany 50: 215-228
Irvine L \& Y Chamberlain. 1994. Seaweeds of the British Isles, Volume 1 Rhodophyta Part 2B Corallinales, Hildenbrandiales, 272 pp. The Natural History Museum, London.

Lara-Domínguez A. 2005. Pastos marinos. En: MorenoCasasola P, E Peresbarbosa-Rojas \& AC Travieso-Bello (eds). Manejo costero integral: el enfoque municipal, pp. 229240. Instituto de Ecología / Comisión Nacional de Áreas Naturales Protegidas, Xalapa.

Leliaert F, W Vanreusel, O De Clerck \& E Coppejans. 2001. Epiphytes on the seagrasses of Zanzibar Island (Tanzania), floristic and ecological aspects. Belgian Journal of Botany 134(1): 3-20.

Littler DS \& MM Littler. 1999. Blade abandonment/ proliferation: a novel mechanism for rapid epiphyte control in marine macrophytes. Ecology 80(5): 1736-1746.

Littler DS \& MM Littler. 2000. Caribbean reef plants. An identification guide to the reef plants of the Caribbean, Bahamas, Florida and Gulf of Mexico, 542 pp. Offshore Graphics, Washington.

Littler MM \& DS Littler. 1980. The evolution of thallus form and survival strategies in benthic marine macroalgae: field and laboratory tests of a functional form model. The American Naturalist 116: 24-44.

López-Calderón J \& R Riosmena-Rodríguez. 2010. Pastos marinos en Laguna San Ignacio, Baja California Sur: Un ecosistema desatendido. CONABIO. Biodiversitas 93: 7-10.

López-Calderón J, R Riosmena-Rodríguez, J Torre \& A Meling-López. 2013. El pasto marino en el Golfo de California: estado actual y amenazas. CONABIO. Biodiversitas 97: 20-15. <http://cobi.org.mx/wp-content/ uploads/2013/02/biodiversitas.pdf >

Magurran A. 1988. Ecological diversity and its measurement, 179 pp. Princeton University Press, New Jersey.

Martínez-Daranas B. 2007. Características y estado de conservación de los pastos marinos en áreas de interés del Archipiélago Sabana-Camagüey, Cuba. Tesis Doctoral, Universidad de La Habana, La Habana, 103 pp.

Mateo-Cid LE \& AC Mendoza-González. 1991. Algas marinas bénticas de la isla Cozumel, Quintana Roo, México. Acta Botanica Mexicana 16: 57-87.

Mateo-Cid LE \& AC Mendoza-González. 2007. Flora ficológica, diversidad, importancia y conservación. En: MejíaOrtiz LM (ed). Biodiversidad acuática de la Isla de Cozumel, pp. 81-113. Universidad de Quintana Roo-Plaza y Valdez, Ciudad de México.

Mateo-Cid LE, AC Mendoza-González \& C Galicia-García. 1996. Algas marinas de Isla Verde, Veracruz, México. Acta Botanica Mexicana 36: 59-65.

Mateo-Cid LE, AC Mendoza-González, R Aguilar-Rosas \& LE Aguilar Rosas. 2006. Algas marinas bentónicas de Puerto Peñasco, Sonora, México. Hidrobiológica 16(3): 4565. 
Mateo-Cid LE, AC Mendoza-González, AG Avila-Ortiz \& S Díaz-Martínez. 2013. Algas marinas bentónicas del litoral de Campeche, México. Acta Botanica Mexicana 104: 5392.

Mateo-Cid LE, I Sánchez-Rodríguez \& E RodríguezMontesinos. 2014. Algas epífitas de Sargassum sinicola Setchell \& Gardner (Fucales, Phaeophyceae), en las islas Magdalena y Margarita en Baja California Sur, México. Revista de Investigaciones Marinas 34(2): 31-44.

Mendoza-González AC \& LE Mateo-Cid. 1992. Algas marinas bentónicas de Isla Mujeres, Quintana Roo, México. Acta Botanica Mexicana 19: 37-61.

Merino M. 1987. The coastal zone of Mexico. Coastal Management 15:27-42.

Montañés M, J Reyes \& M Sansón. 2003. La comunidad de epifitos de Zonaria tournefortiien el norte de Tenerife (Islas Canarias): análisis florístico y comentarios sobre su epifauna. Vieraea 31: 121-132.

Okolodkov YB, JA Aké-Castillo \& C Galicia-García. 2012. Productores primarios acuáticos del Parque Nacional Sistema Arrecifal Veracruzano, golfo de México: estado del conocimiento. En: Sánchez AJ, X Chiappa-Carrara \& R Brito-Pérez (eds). Recursos costeros del sureste, pp. 137158. Consejo de Ciencia, Innovación y Tecnología del Estado de Yucatán, Mérida.

Ortuño-Aguirre C \& R Riosmena-Rodríguez. 2007. Dinámica del epifitismo en Padina concrescens (Dictyotales, Phaeophyta) en el sureste de la Península de Baja California, México. Ciencias Marinas 33: 311-317.

O'Shanahan L, E Vela \& A Sánchez. 2003. Effects of a sewage effluent on a benthic community in the coast of Telde, NE of Gran Canaria (Canary Islands). VIERAEA 31: 253266.

Pielou E. 1975. Ecological diversity, 165 pp. John Wiley \& Sons, New York.

Quan-Young L, M Díaz-Martín \& J Espinoza-Avalos. 2006. Algas epífitas de Bajo Pepito, Isla Mujeres, Quintana Roo, México. Revista de Biología Tropical 54(2): 317-328.

Robinson NM, C Galicia-García \& YB Okolodkov. 2012. New records of Green (Chlorophyta) and Brown algae (Phaeophyta) for Cabezo reef, National Park Sistema Arrecifal Veracruzano, Gulf of Mexico. Acta Botanica Mexicana 101: 11-48.

Sánchez-Lizaso JL \& R Riosmena-Rodríguez. 1997. Macroalgas epífitas de Zostera marina L. en Bahía Concepción, B.C.S., México. Oceánides 12(1): 55-59.

Sand-Jensen K \& J Borum. 1991. Interactions among phytoplankton, periphyton and macrophytes in temperate freshwaters and estuaries. Aquatic Botany 41: 137-175.

Saunders JE, MJ Attrill, AM Shaw \& AA Rowden. 2003. Spatial variability in the epiphytic algal assemblages of Zostera marina seagrass beds. Marine Ecology Progress Series 249: 107-115.
Schneider C \& R Searles. 1991. Seaweeds of the southeastern United States, Cape Hatteras to Cape Cañaveral, 563 pp. Duke University Press, Durham.

Sebens KP. 1986. Spatial relationship among encrusting marine organisms in the New England subtitle zone. Ecological Monographs 56: 73-96.

Sepúlveda-Lozada A. 2004. Epificoflora estacional de Thalassia testudinum Banks ex König (Hydrocharitaceae), en la llanura arrecifal de la Isla de Sacrificios, Ver., México. Tesis Profesional, Facultad de Biología, Universidad Veracruzana, Xalapa, Veracruz, 85 pp.

Short FT \& DM Burdick. 1996. Quantifying eelgrass habitat loss in relation to housing development and nitrogen loading in Waquoit Bay. Massachusetts. Estuaries 19: 730-739.

Sistema Meteorológico Nacional. 2017. Resúmenes mensuales de temperaturas y lluvia. Servicio Meteorológico Nacional, Comisión Nacional del Agua (CONAGUA), Secretaría de Medio Ambiente y Recursos Naturales (SEMARNAT), Ciudad de México. <http://smn.conagua. gob.mx/

Spalding M, M Taylor, C Ravilious, F Short \& E Green. 2003. Global overview. The distribution and status of seagrasses. In: Green EP \& FT Short (eds). World atlas of seagrasses, pp. 5-26. UNEP World Conservation Monitoring Centre, University of California Press, Berkeley.

Steneck RS. 1986. The ecology of coralline algal crusts: Convergent patterns and adaptative strategies. Annual Review of Ecology, Evolution and Systematics 17: 273-303.

Steneck RS \& M Dethier. 1994. A functional group approach to the structure of algal dominated communities. Oikos 69 : 476-498.

Sureda A, B Antonio, T Jorge, S Deudero \& P Antoni. 2008. Antioxidant response of the seagrass Posidonia oceanica when epiphytized by the invasive Macroalgae Lophocladia lallemandii. Marine Environmental Research 66:359-363.

Széchy MTM \& ADF Sá. 2008. Variacão sazonal do epifitismo por macroalgas em una populacão de Sargassum vulgare C. Agardh (Phaeophyceae, Fucales) da Bahia da Ilha Grande, Rio de Janeiro. Oecologia Brasiliensis 12: 299-314.

Taylor W. 1960. Marine algae of the Eastern Tropical and Subtropical Coasts of the Americas, 870 pp. University of Michigan Press, Ann Arbor.

Terrados J \& J Borum. 2004. Why are seagrasses important?Goods and services provided by seagrass meadows. In: Borum J, CM Duarte, D Krause-Jensen \& T Greve (eds). European seagrasses: An introduction to monitoring and management, pp. 8-10. The M\&MS Project, Copenhagen.

Valiela I, J McClelland, J Hauxwell, P Behr, D Hersh \& K Foreman. 1997. Macroalgal blooms in shallow estuaries: controls and ecophysiological and ecosystem consequences. Limnology and Oceanography 42: 1105-1118. 
van den Hoek C. 1982. A taxonomic revision of the American species of Cladophora (Chlorophyceae) in the North Atlantic Ocean and their geographic distribution, $236 \mathrm{pp}$. North-Holland Publishing Company, Amsterdam.

van Montfrans J, RL Wetzel \& RJ Orth. 1984. Epiphytegrazer relationships in seagrass meadows: consequences for seagrass growth and production. Estuaries 7: 289-309.

van Tussenbroek BI, MG Barba, JGR Wong, JK van Dijk \& M Waycott. 2010. Guía de los pastos marinos tropicales del Atlántico oeste, 75 pp. Instituto de Ciencias del Mar y Limnología, Ciudad de México.

Wan AHL, RJ Wilkes, S Heesch, R Bermejo, MP Johnson \& L Morrison. 2017. Assessment and characterisation of Ireland's green tides (Ulva species). PLoS ONE 12(1): e0169049 <doi:10.1371/journal.pone.0169049>.

Wear DJ, MJ Sullivan, AD Moore \& DF Millie. 1999. Effects of water-column enrichment on the production dynamics of three seagrass species and their epiphytic algae. Marine Ecology Progress Series 179: 201-213.
Wilkinson T, E Wiken, J Bezaury-Creel, T Hourigan, T Agardy, H Herrmann, L Janishevski, C Madden, L Morgan \& M Padilla. 2009. Ecorregiones marinas de América del Norte, 200 pp. Comisión para la Cooperación Ambiental, Montreal.

Won B, T Cho \& S Fredericq. 2009. Morphological and molecular characterization of species of the genus Centroceras (Ceramiaceae, Ceramiales), including two new species. Journal of Phycology 45: 227-250.

Wynne MJ. 2011. A checklist of benthic marine algae of the tropical and subtropical western Atlantic: third revision. Nova Hedwigia Beihefte 140(1-6): 7-66.

Zieman JC. 1982. The ecology of the Seagrasses of south Florida: A community profile, 158 pp. U.S.A. Fish and Wildlife Services, Office of Biological Services, Washington DC. 